\title{
TASTE ALTERATION AND QUALITY OF LIFE OF PATIENTS RECEIVING CHEMOTHERAPY
}

\section{ANOOJA ANTONY, SHEELA PAVITHRAN*}

Department of Medical Surgical Nursing, Amrita College of Nursing, Amrita University, Kochi, India. Email: sheelapavithran@aims.amrita.edu Received: 30 June 2017, Revised and Accepted: 21 September 2017

\begin{abstract}
Objective: The study was conducted with the aim to determine the taste alteration (TA) and its relation with quality of life (QOL) of patients receiving chemotherapy.
\end{abstract}

Methods: The present study was undertaken among 100 patients receiving cancer chemotherapy with the aim of identifying the commonly experienced TA and its relation with QOL of the patients. The patients were selected by convenience sampling from a selected Cancer Institute in Kerala.

Results: The study reported that TA was present in all the patients receiving chemotherapy ranging from mild to severe where $50 \%$ had moderate and $9 \%$ had severe alteration in taste. There were 19\% patients who had poor and $45 \%$ who had average QOL. The TAs had a moderately negative correlation with QOL ( $\mathrm{r}=-0.51$ ) indicating that an increase in TA decreases the QOL.

Conclusion: The study evinces that TA is a common side effect of chemotherapy which often impacts the QOL negatively. Hence, it is essential to understand the types of TA and the specific drug causing it. This will enable the health-care team members to develop treatments for these conditions and educate patients regarding the strategies to be adopted to manage the problem, thereby improving patients' QOL.

Keywords: Chemotherapy, Taste alterations, Quality of life, Chemotherapy-induced taste alteration scale, Side effects of chemotherapy.

(C) 2017 The Authors. Published by Innovare Academic Sciences Pvt Ltd. This is an open access article under the CC BY license (http://creativecommons. org/licenses/by/4. 0/) DOI: http://dx.doi.org/10.22159/ajpcr.2017.v10i12.21049

\section{INTRODUCTION}

Cancer has been the second main cause of death in India. According to the World Health Organization, cancers figure among the leading cause of morbidity and mortality worldwide, with approximately 14 million new cases and 8.2 million cancer-related deaths in 2012. The number of new cases is expected to rise by about $70 \%$ over the next two decades [1]. As the incidence of cancer is increasing, the new modalities of treatments have also become more significant [2].

Cancer treatments mainly include radiation therapy, surgery, and chemotherapy. Chemotherapy is one of the primary therapeutic regimens for treating cancer $[2,3]$. Common side effects of chemotherapy include fatigue, alopecia, anemia, easy bruising, diarrhea, constipation, alteration in taste, and appetite. Taste alteration (TA) is the most distressing side effect but frequently unrecognized problem which has a serious impact on the nutrition and QOL of the patients [4]. The exact cause of alteration in taste in these patients is unknown. Distorted senses may also produce a metallic or bitter taste or impair the patient's ability to detect certain odors. Alterations in the sense of taste may also affect the ability to taste many foods [5]. It is believed that tastants and chemicals in foods are detected by the taste buds, which consists of special sensory cells and when stimulated, these cells send signals to specific areas of the brain, which makes one conscious of taste [6]. The TA, adversely affecting the life quality of individuals, should be evaluated in a comprehensive manner for effective and appropriate management of the symptoms [7].

Bernhardson et al. who conducted a qualitative study on chemosensory changes experienced by 21 patients undergoing cancer chemotherapy in Sweden reported about TA, but the information regarding this is poorly understood [7]. This poor understanding combined with a lack of adequate knowledge on TAs may indicate that it is particularly needed to study on TAs among patients receiving chemotherapy, but it may be challenging for the health-care team members to discuss these problems with the patients [8].
In daily clinical routine, patients rarely addressed TAs spontaneously and even physicians often consider them as a side effect that is unavoidable. TAs were alarmingly underrecognized by caregivers as of 1998 [5], and the situation has improved little science. Medical treatment options regarding TAs have also not been thoroughly investigated [9]. Moreover, TAs are not given enough attention today in clinical decision-making, and the Indian literature regarding the TA after chemotherapy is scarce. Neither the investigators have come across with similar literature in the state of Kerala.

The study would help to estimate the magnitude or the severity of the TA among patients receiving chemotherapy which seriously impact the ability to eat or even taste the food. This will in turn affect the quality of life (QOL) of the patient to such an extent that they may discontinue the treatment at any point of time. Since no much study is conducted in this area or inadequacy of literature, the findings of this study would be an eye opener for future research in this area.

A more thorough knowledge of taste complaints and possible interventions may impact the outcome of cancer therapy, reduce the cost of care, and improve the QOL of the patients. A better understanding of the onset of the mechanism of taste changes would allow the clinicians to intervene earlier in the course of therapy before such changes have a negative impact on nutrition and QOL.

\section{METHODS}

The descriptive correlational survey was used for the study with the aim to determine the status of taste among patients receiving chemotherapy and their QOL and also to find the correlation between the TA and QOL. The study was undertaken among 100 patients receiving chemotherapy selected by convenience sampling. The setting selected was a Cancer Institute of a selected Multi Specialty Hospital in Kerala, South India.

Patients between the age of 20 and 65 years and who had received at least three cycles of chemotherapy (as per the review of the medical 
record) were included in the study. As part of the ethical consideration, ethical clearance from the Institutional Ethical Committee and informed consent from each of the subjects were obtained. Sociodemographic and clinical data were collected using semi-structured questionnaire developed by the researcher, chemotherapy-induced TA scale (CiTAS) [10] to assess the status of taste, and University of Washington QOL questionnaire [11] to assess the QOL of patients. Subjects took approximately 30 minutes to complete the questionnaire.

\section{RESULTS}

The data were analyzed using frequency, percentage, and also independent $\mathrm{t}$-test.

\section{Socio-demographic data}

The mean age of the subjects was $52.5 \pm 10.6$ years. Males were $61 \%$, married (79\%), and 54\% were educated up to intermediate/post-high school and unemployed. There were $17 \%$ who were smokers (currently stopped) and $21 \%$ who use alcohol, and none of them had the habit of chewing tobacco or use of illicit drugs.

\section{Clinical data}

The family history of cancer was reported in $13 \%$ of the patients. Data also indicated that $45(45 \%)$ of subjects were in Stage IV of disease followed by 39 (39\%) in Stage III. With regard to the treatment modality, $64(64 \%)$ followed chemotherapy and $61(61 \%)$ followed combination chemotherapy and maximum number of patients $32(32 \%)$ were in the fourth cycle of chemotherapy. Comorbidities were present in $22 \%$, and out of which majority, i.e. 14 (63\%) had hypertension and 7 (13\%) had diabetes mellitus.

With regard to brushing practices, almost all (99\%) used paste for brushing, all the subjects brushed teeth twice daily, 16 (16\%) used dentures, and $81(81 \%)$ used tongue cleaner for cleaning the tongue. Majority of the patients $86(86 \%)$ were non-vegetarians and $63(63 \%)$ had normal body mass index, while 24 (24\%) were overweight.

Fig. 1 indicates that breast cancer outnumbered $(25,25 \%)$ other cancers such as carcinoma colon (12\%), rectum (10\%), and lungs (9\%).

Persistent dry mouth (60\%), pain or soreness in the mouth $(42 \%)$, and dental cavities or dental caries 38 (38\%) were the main problems experienced by the patients. Frequent nasal congestion was reported by $21(21 \%)$ and cold or flu longer than a month by $15(15 \%)$ of the subjects (Table 1).

\section{Alteration in taste}

Half of the subjects $(n=50)$ had moderate, and $9(9 \%)$ had a severe alteration in taste. There is no one who expressed that they do not have TAs (Fig.2).

Table 2 presents item-wise distribution of alteration in taste among patients receiving chemotherapy. The item-wise analysis indicates that 31 (31\%) of the subjects reported that they have difficulty to taste food, i.e., they were "unable to taste at all." With regard to different flavors, the greatest difficulty was encountered with bitter taste where $23(23 \%)$ were "unable to taste at all" the bitter taste and $26(26 \%)$ reported "quite difficult to taste" which altogether constituted 49 (49\%). A total of $41(41 \%)$ each had difficulty in tasting saltiness and sourness. Further, it is noted that $15(15 \%)$ were unable to "taste at all" the saltiness and $26(26 \%)$ for whom it was "quite difficult to taste" saltiness. Similarly, $12(12 \%)$ were "unable to taste at all" the sourness and $29(29 \%)$ for whom it was "quite difficult to taste" sourness. With regard to the subscale "unpleasant taste change," 42 (42\%) did not perceive unpleasant taste change in smell and flavor, whereas 61 (61\%) reported that food does not taste as it should be, i.e., 38\% very unpleasant and $23 \%$ quite unpleasant, and 46 (46\%) reported that they had bitter taste in the mouth (very unpleasant - $26 \%$ and quite unpleasant - 20\%). With regard to the subscale, "unpleasant symptoms or problems," "feeling nauseated and queasy" was the most unpleasant symptom (where 10\%
Table 1: Clinical problems of subjects receiving chemotherapy $\mathrm{n}=\mathbf{1 0 0}$

\begin{tabular}{ll}
\hline Clinical problems & Frequency (\%) \\
\hline Persistent dry mouth & $60(60)$ \\
Pain or soreness in any part of the mouth & $42(42)$ \\
Dental cavities or dental caries & $38(38)$ \\
Frequent nasal congestion from allergies & $21(21)$ \\
Cold or flu for longer than a month & $15(15)$ \\
Undergone any dental procedures recently & $4(4)$ \\
History of nasal polyps & $4(4)$ \\
Sinus infections & $2(2)$ \\
History of head injury & $1(1)$ \\
Broken nose or any serious injury to face and & $0(0)$ \\
skull & \\
History of head and neck surgery & $0(0)$ \\
\hline
\end{tabular}

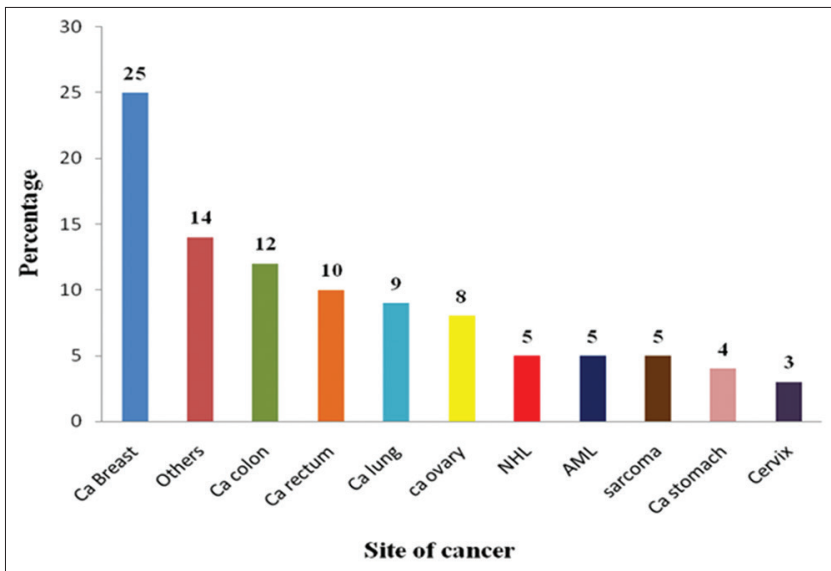

Fig. 1: Bar diagram showing the site of cancer among subjects receiving chemotherapy

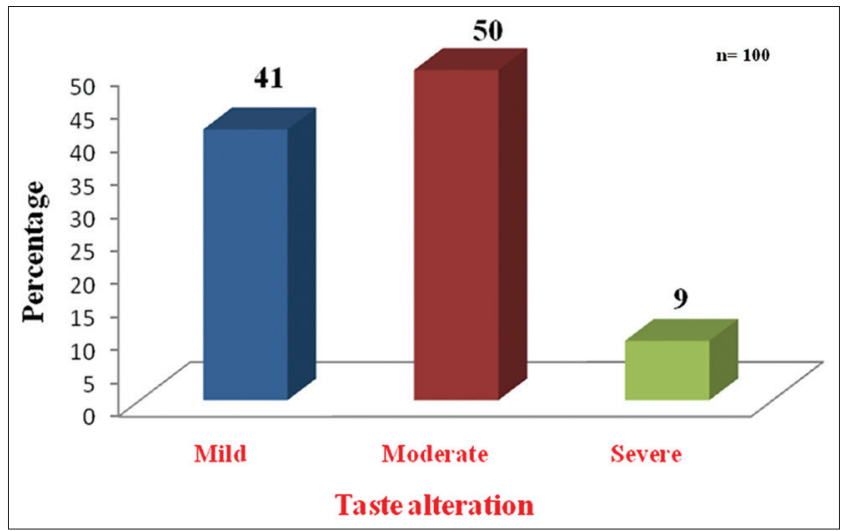

Fig. 2: Bar diagram showing taste alteration among patients receiving chemotherapy

felt "very nauseated" and 25\% "quite nauseated"). At the same time, $83(83 \%)$ reported that they do not have difficulty in eating food and $78(78 \%)$ each reported that they do not have difficulty in eating oily food and meat.

A description of subscales of the TA is presented in Table 3 that indicates that the mean change in the sense of taste is $16.97 \pm 8.092$, unpleasant taste change is $15.84 \pm 7.166$, and unpleasant symptoms $11.22 \pm 4.651$.

QOL

$19 \%$ of the subjects had poor QOL and 45\% with average QOL. 
Table 2: Status of TAs among patients receiving chemotherapy $n=100$

\begin{tabular}{|c|c|c|c|c|c|}
\hline TAs & Taste normally & $\begin{array}{l}\text { Slightly difficult } \\
\text { to taste }\end{array}$ & $\begin{array}{l}\text { Somewhat difficult } \\
\text { to taste }\end{array}$ & $\begin{array}{l}\text { Quite difficult } \\
\text { to taste }\end{array}$ & $\begin{array}{l}\text { Unable to } \\
\text { taste at all }\end{array}$ \\
\hline \multicolumn{6}{|l|}{ Change in the Sense of Taste } \\
\hline Have difficulty tasting food & 19 & 11 & 10 & 29 & 31 \\
\hline Have difficulty tasting sweetness & 45 & 8 & 14 & 23 & 10 \\
\hline Have difficulty tasting saltiness & 34 & 5 & 20 & 26 & 15 \\
\hline Have difficulty tasting sourness & 36 & 7 & 16 & 29 & 12 \\
\hline Have difficulty tasting bitterness & 32 & 7 & 12 & 26 & 23 \\
\hline Unpleasant Taste Change & No & Slightly & Somewhat & Quite & Very \\
\hline Unable to perceive the smell or flavor of food & No & Slightly & Somewhat & Quite & Very \\
\hline Everything tastes bad & 61 & 4 & 15 & 16 & 4 \\
\hline Food does not taste as it should & 22 & 6 & 11 & 23 & 38 \\
\hline Have a bitter taste in the mouth & 35 & 12 & 7 & 20 & 26 \\
\hline Have a bad taste in the mouth & 41 & 6 & 25 & 21 & 7 \\
\hline Everything tastes bitter & 45 & 6 & 12 & 14 & 23 \\
\hline Unpleasant Symptoms or Problems & No & Slightly & Somewhat & Quite & Very \\
\hline Bothered by the smell of food & 49 & 8 & 20 & 19 & 4 \\
\hline Have difficulty eating hot food & 83 & 5 & 5 & 7 & 0 \\
\hline Have difficulty eating oily food & 78 & 12 & 6 & 4 & 0 \\
\hline Have difficulty eating meat & 78 & 9 & 6 & 5 & 2 \\
\hline Have a reduced appetite & 46 & 8 & 15 & 26 & 5 \\
\hline
\end{tabular}

TAs: Taste alterations

Table 3: Description of taste alteration based on subscale $\mathbf{n = 1 0 0}$

\begin{tabular}{ll}
\hline Subscales & Mean \pm SD \\
\hline Change in the sense of taste & $16.97 \pm 8.092$ \\
Unpleasant taste change & $15.84 \pm 7.166$ \\
Unpleasant symptoms & $11.22 \pm 4.651$ \\
Total & $44.03 \pm 18.34$ \\
\hline
\end{tabular}

SD: Standard deviation, TAs: Taste alterations

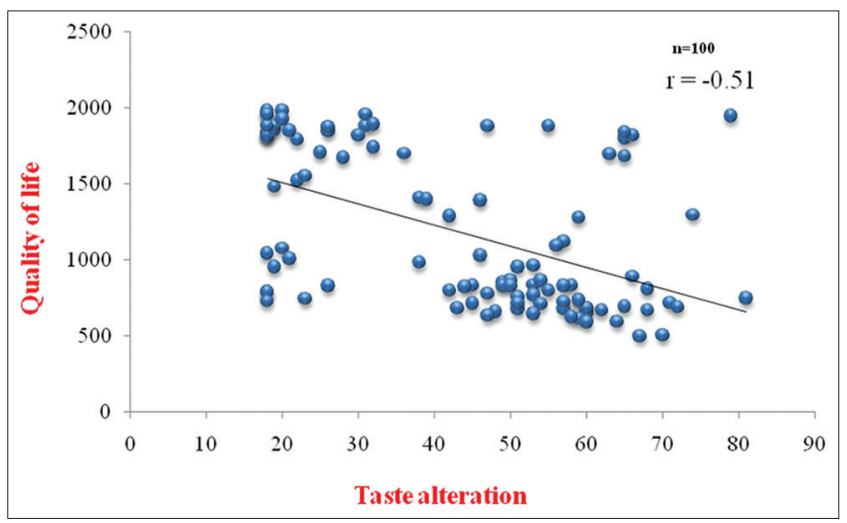

Fig. 3: Scatter diagram showing correlation between quality of life and taste alteration among patients receiving chemotherapy

A moderate negative correlation exists between TA and QOL ( $\mathrm{r}=-0.51$ ) which can be interpreted that, as TA increases, QOL has a tendency to decrease (Fig. 3).

The t-value computed between TA and contributing factors indicated that the factors such as cold or flu longer than a month $\left(t_{(98)=} 2.42\right)$; persistent dry mouth $\left(\mathrm{t}_{(98)}=4.87\right)$; frequent nasal congestion from allergies $\left(\mathrm{t}_{(98)}=2.96\right)$; pain or soreness in any part of the mouth $\left(\mathrm{t}_{(98)}=5.88\right)$; dental cavities or dental caries $\left(\mathrm{t}_{(98)=} 3.55\right)$, and $\mathrm{p}<0.05$ significantly contributed to TA among patients receiving chemotherapy.

\section{DISCUSSION}

The investigator had come across with only very few studies related to TA among patients receiving chemotherapy. Unfortunately, the investigator did not come across with studies conducted in India or
Kerala. The main aim of the study was to determine the status of TA, QOL, and the relation of TA with QOL and contributing factors of TA.

The present study reported that $50(50 \%)$ of the patients receiving chemotherapy had moderate and 9 (9\%) had a severe alteration in taste. It is worth noticing that TA was present in all the subjects receiving chemotherapy from mild to severe form.

The study findings are supported by Zabernigg et al. who reported that TA was present in $69.9 \%$ subjects out of 197 cancer patients who received chemotherapy [12]. In the present study, 59\% of patients had average-to-severe TA. In comparing, the present study with the reported studies, it can be interpreted that the prevalence of TA occurs in patients receiving chemotherapy which ranged from 40 to $70 \%$.

The results of the present study regarding QOL of patients receiving chemotherapy showed that among the 100 subjects receiving chemotherapy, 19 (19\%) of subjects had poor QOL, and 45 (45\%) had an average QOL.

Hutton et al. reported that chemosensory dysfunction is a primary factor in the evolution of declining nutritional status and QOL in patients with advanced cancer among 66 patients with advanced cancer who were receiving palliative care. Only $14 \%$ of the subjects reported no chemosensory complaints of any kind, whereas $86 \%$ reported some degree of chemosensory abnormalities [13]. On comparing the study results with the abovementioned study, it is evident that the TAs affect the QOL of patients. The present study reported that $45 \%$ of the subjects had average and $19 \%$ had poor QOL.

In the present study, also a moderately negative correlation exists between TA and QOL $(\mathrm{r}=-0.51)$. Even though the study does not provide a strong correlation between TAs and QOL, this study favors the argument that TAs negatively impact the QOL. The study might have shown strong correlation if the sample size was much higher. However, the study has not attempted to study the effect of the disease on TAs which may contribute to QOL of patients.

A study conducted by Zabernigg et al. on TAs in cancer patients receiving chemotherapy reported that TAs were significantly associated with a reduction in various aspects of QOL. The strongest correlations found for TAs were with appetite loss $(\mathrm{r}=-0.39)$, fatigue $(\mathrm{r}=-0.40)$, nausea/ 
vomiting ( $\mathrm{r}=-0.35)$, and cognitive functioning $(\mathrm{r}=-0.37)$. Correlations between TAs and all other EORTC QLQC 30 scales were $<0.35$. All correlations were statistically significant at $\mathrm{p}<0.001$ [12]. The present study has a comparatively higher $r$ value than the reported study ( $r=0.51$ vs. $r$ ranging from 0.35 to 0.40 ).

Out of curiosity, the researcher tried to find the association between QOL and selected clinical variables. The present study failed to identify any association between the treatment modalities undergone, type of chemotherapy regimen, and number of cycles of chemotherapy ( $p>0.05)$. The most common complaints were persistent bad taste in the mouth, taste distortion, and heightened sensitivity to odors which in turn affect the patient's QOL.

Rehwaldt et al. who conducted a study among 42 cancer patients who had received at least two cycles of chemotherapy at oncology center in Illinois reported that most of the patients who had changes in their taste had affected their ability to eat. Taste changes and strategies varied somewhat according to chemotherapy regimen [14]. Nurses have a specific role in educating the patients which will help them to anticipate taste changes and follow-up should focus on self-care to cope with actual taste change perceptions.

\section{CONCLUSION}

The findings of the present study indicate that TAs are commonly experienced by patients receiving chemotherapy, even though its severity varies from patient to patient and probably with different chemotherapeutic agents, modalities of treatment [15], and disease itself. The TAs along with the malignancy itself affect the QOL [16-18]. Since TA is a distressing side effect of chemotherapy and can greatly affect the nutrition of the patients, great care must be taken from the initiation of the therapy to prevent the progression of the distress.

The present study provides an insight to the nurse about chemotherapyinduced TA and the role she plays in identifying, reporting, and managing the same. It stresses about the importance of patient education regarding the self-care measures to be adopted. Further studies need to be conducted to suggest various ways of managing symptoms which help the patients to improve their alterations in taste and QOL.

The study has not determined the influence of comorbidities in TAs. The literature supports that cancer/malignancies can affect TAs, but that aspect is not investigated in this study which could have influenced the outcome. The study included patients receiving different regimens and not a single regimen which could also have an effect on the outcome of the study.

\section{ACKNOWLEDGMENT}

The authors would like to thank Dr. Taro Kano RN, PhD and Dr. Rogers SN for providing the permission to use the tool CiTAS and UWQOL, respectively. The authors thank all the participants for their wholehearted participation.

\section{REFERENCES}

1. World Health Organization. WHO Media Centre; 2015. Available from: http://www.who.int/mediacentre/factsheets/fs297/en. [Last cited on $2015 \mathrm{Feb}]$.

2. Neelakandan K, Babu P, Nair S. Emerging roles for modulation of microRNA signatures in cancer chemoprevention. Curr Cancer Drug Targets 2012;12(6):716-40

3. Comeau TB, Epstein JB, Migas C. Taste and smell dysfunction in patients receiving chemotherapy: A review of current knowledge. Support Care Cancer 2001;9(8):575-80

4. Drisya PM, James E. Recent updates in the management of chemotherapy induced nausea and vomiting. Asian J Pharm Clin Res 2013;6:5-10

5. Osborn HT, Akoh CC. Effects of natural antioxidants on iron catalysed lipid oxidation of structured lipid base emulsions. J Am Oil Chem Soc 2003;80:847-52.

6. Baltimore, MD. Society for Neurosciences. Available from http:// www.brainfacts.org/sensing-thinkig-behaving/learning-and-memory/ articles/2012/different-facets-of-memory.

7. Bernhardson BM, Tishelman C, Rutqvist LE. Chemosensory changes experienced by patients undergoing cancer chemotherapy: A qualitative interview study. J Pain Symptom Manage 2007;34(4):403-12.

8. Newell S, Sanson-Fisher RW, Girgis A, Bonaventura A. How well do medical oncologists' perceptions reflect their patients' reported physical and psychosocial problems? Data from a survey of five oncologists. Cancer 1998;83(8):1640-51.

9. Strasser F, Demmer R, Böhme C, Schmitz SF, Thuerlimann B, Cerny T, et al. Prevention of docetaxel-or paclitaxel-associated taste alterations in cancer patients with oral glutamine: A randomized, placebocontrolled, double-blind study. Oncologist 2008;13(3):337-46.

10. Kano T, Kanda K. Development and validation of a chemotherapyinduced taste alteration scale. Oncol Nurs Forum 2013;40(2):E79-85.

11. Rogers SN, Lowe D. Screening for dysfunction to promote multidisciplinary intervention by using the University of Washington Quality of Life Questionnaire. Arch Otolaryngol Head Neck Surg 2009;135(4):369-75.

12. Zabernigg A, Gamper EM, Giesinger JM, Rumpold G, Kemmler G, Gattringer $\mathrm{K}$, et al. Taste alterations in cancer patients receiving chemotherapy: A neglected side effect? Oncologist 2010;15(8):913-20.

13. Hutton JL, Baracos VE, Wismer WV. Chemosensory dysfunction is a primary factor in the evolution of declining nutritional status and quality of life in patients with advanced cancer. J Pain Symptom Manage 2007;33(2):156-65.

14. Bernhardson BM, Tishelman C, Rutqvist LE. Self-reported taste and smell changes during cancer chemotherapy. Support Care Cancer 2008;16(3):275-83.

15. Indumathi P, Shanmuga PE. Incidence and management of treatmentrelated side effects for patients receiving platinum therapy in an outpatient oncology clinic. Asian J Pharm Clin Res 2017;10:117-20

16. Trivedi NP, Swaminathan DK, Thankappan K, Chatni S, Kuriakose MA, Iyer S. Comparison of quality of life in advanced laryngeal cancer patients after concurrent chemoradiotherapy versus total laryngectomy. Otolaryngol Head Neck Surg 2008;139:702-7.

17. Kirthi C, Afzal A, Reddy M, Ali SA, Yerramilli A, Sharma S. A study on the adverse effects of anticancer drugs in an oncology center of a tertiary care hospital. Int J Pharm Pharm Sci 2014;6(2):580-3.

18. Noviyani R, Indrayathi PA, Budiana IN, Suwiyoga K, Tunas K. Assessment of life quality in patients with Stage IIB-IIIB squamous cell cervical cancer receiving paclitaxel cisplatin chemotherapy regimen by EORTC QLQ-C30 questionnaire in sanglah Hospital Denpasar. Int J Pharm Pharm Sci 2017;9:222-6. 